\title{
Determinants of early outcome after neonatal cardiac surgery in a developing country
}

Kinjal D. Bakshi, FNB, a Balu Vaidyanathan, DM, ${ }^{a}$ Karimassery R. Sundaram, PhD, ${ }^{\text {b }}$ Stephen J. Roth, MD, MPH, Krishnanaik Shivaprakasha, $\mathrm{McH}^{\mathrm{c}}{ }^{\mathrm{C}}$ Suresh G. Rao, $\mathrm{McH}^{\mathrm{c}}$ Suresh G. Nair, MD, ${ }^{\mathrm{d}}$ Suresh Chengode, MD, ${ }^{d}$ and R. Krishna Kumar, DM, FACC

From the Departments of Pediatric Cardiology, ${ }^{\text {a }}$ Biostatistics, ${ }^{\text {b }}$ Pediatric Cardiac Surgery, ${ }^{\mathrm{c}}$ and Cardiac Anesthesia, ${ }^{\mathrm{d}}$ Amrita Institute of Medical Sciences and Research Center, Kerala, India; and the Department of Pediatrics, ${ }^{\text {e }}$ Stanford University School of Medicine, and Lucile Packard Children's Hospital, Palo Alto, Calif.

Received for publication March 19, 2007; revisions received April 12, 2007; accepted for publication April 23, 2007.

Address for reprints: Balu Vaidyanathan, MD, DNB, (Pediatrics), DM (Cardiology), Pediatric Cardiology, Amrita Institute of Medical sciences, Elamakkara P.O., Kochi, Kerala, India Pin: 682026 (E-mail: baluvaidyanathan@ aims.amrita.edu, baluvaidyanathan@gmail. com).

J Thorac Cardiovasc Surg 2007;134:765-71 $0022-5223 / \$ 32.00$

Copyright $(9) 2007$ by The American Association for Thoracic Surgery

doi:10.1016/j.jtcvs.2007.04.042
Objective: Significant technologic advances have improved outcomes in neonatal cardiac surgery over the past 3 decades. However, outcomes might be different in developing countries with resource limitations. We sought to identify the determinants of early outcome after neonatal cardiac surgery in a tertiary referral center in South India.

Methods: Hospital records of 330 consecutive neonates who underwent surgical intervention between January 1999 and April 2006 were reviewed, and perioperative variables were recorded. Main outcome measures were 30-day mortality, postoperative bloodstream infection, and hospital stay of longer than 10 days. Multivariate logistic regression analysis was performed.

Results: Overall mortality was $8.8 \%$. Mortality significantly decreased from $21.4 \%$ before 2002 to $4.3 \%$ after 2002 (3.2\% for corrective operations, $P<.0001)$. The prevalence of postoperative bloodstream infection remained the same, whereas surgical site infection and hospital stay significantly increased after 2002. Predictors of outcomes on multivariate analysis were as follows: (1) mortality-operation before 2002 (odds ratio, 5.5), age less than 7 days (odds ratio, 3.8), preoperative antibiotic use (odds ratio, 5.6), and postoperative exchange transfusion (odds ratio, 14.9); (2) postoperative bloodstream infection (21.2\%) - use of cardiopulmonary bypass (odds ratio, 2.0), reintubation (odds ratio, 7.7), and surgical site infection (odds ratio, 4.1); and (3) hospital stay of longer than 10 days (61.2\%) - use of cardiopulmonary bypass (odds ratio, 2.8), delayed sternal closure (odds ratio, 3.6), reintubation (odds ratio, 12.1), surgical site infection (odds ratio, 13.8), and postoperative antibiotic use (odds ratio, 4.4).

Conclusions: With increasing experience, neonatal cardiac surgery can be performed with excellent outcomes in developing countries with resource limitations. Infectious complications contribute significantly to morbidity and mortality, and improvements in infection-control practices should be emphasized to improve outcomes further.

$\mathrm{T}$ The field of pediatric and neonatal cardiac surgery has witnessed major advances in the last 30 years. ${ }^{1-3}$ In the developed world there is a strong emphasis on performing corrective operations early in life, often in the neonatal period itself, for forms of congenital heart disease (CHD) that can be corrected. ${ }^{4,5}$ Most centers in the developed world have reported excellent outcomes for neonatal cardiac surgery, with in-hospital mortality of less than $5 \% .^{6-11}$ This has become possible because of technologic refinements, improved multidisciplinary expertise, and advances in the understanding of the pathophysiology and natural history of CHD. ${ }^{12}$ Most developed countries have well-established systems for diagnosis and referral of sick newborns with CHD. Centers performing neonatal 


\section{Abbreviations and Acronyms \\ $\mathrm{CHD}=$ congenital heart disease \\ $\mathrm{CPB}=$ cardiopulmonary bypass \\ $\mathrm{ICU}=$ intensive care unit}

cardiac surgery have dedicated pediatric cardiac programs with sophisticated infrastructure and focused expertise in the various aspects of pediatric cardiac care. Many centers have the capability to perform extracorporeal circulatory support, which has further improved survival, especially for the highest-risk patients. ${ }^{13}$ With decreasing in-hospital mortality, the focus has shifted toward long-term outcomes and especially neurodevelopmental sequelae. ${ }^{14,15}$

However, the situation in the developing world is strikingly different. Congenital cardiac abnormalities are the second most important cause of childhood mortality in high-income countries but rank only sixth or lower in middle- and low-income countries. ${ }^{16}$ Delayed recognition of CHD is common, and most critical CHD is detected after newborns are discharged from the hospital. The problem is compounded by the lack of a structured system for referral and transport of sick neonates with suspected CHD to a tertiary cardiac care center. As a result, most newborns present to referral centers in a suboptimal preoperative state, and significant attrition occurs before hospital presentation. ${ }^{17}$ Most centers in developing countries, such as India, work with the inherent limitations in trained manpower, infrastructure, and equipment for postoperative care that accompany fewer economic resources. ${ }^{16}$ Furthermore, significant financial constraints are imposed by the absence of health insurance coverage. All of these factors present a significant challenge for the surgical management of neonates with CHD. Despite these limitations, a growing number of centers in the developing world have started performing corrective operations for neonates with critical CHD in the past decade. ${ }^{18,19}$

In this study we report the early results and an analysis of the determinants of outcomes of corrective and palliative neonatal cardiac surgery in 330 patients in a tertiary care pediatric cardiac program in the State of Kerala in southern India.

\section{Materials and Methods \\ Type of Study}

This was a retrospective analysis of the hospital records of all neonates (age, $<30$ days) who underwent surgical intervention for $\mathrm{CHD}$ at our center during the period from January 1999 through April 2006.

\section{Study Setting}

The data were obtained from a tertiary care pediatric cardiac facility that caters to a population of approximately 30 million in the State of Kerala in southern India. The program started in 1999 with significant limitations in physician and nursing staff and intensive care beds. Over the next 4 years, efforts were made to improve the infrastructure and manpower. Additional personnel were recruited, and a separate step-down intensive care unit (ICU) was created. Collaboration with a well-established pediatric cardiovascular program (Children's Hospital Boston) was obtained to improve standards of nursing care and establish ICU protocols. There were no formal attempts to establish systems for neonatal transport in the region because of economic limitations, and few prenatally diagnosed patients were transported in utero during the study period. Extracorporeal membrane oxygenation and ventricular assist devices were not available. Although all personnel were encouraged to follow standardized infection-control guidelines, ${ }^{20}$ attempts to establish formal infection-control systems were only made toward the end of the study period.

\section{Patients}

All neonates (age, $<30$ days) who underwent corrective and palliative operations for $\mathrm{CHD}$ at our center during the study period were included. Surgical intervention for hypoplastic left heart syndrome and severe Ebstein anomaly was not performed. The diagnosis of CHD was established by means of echocardiography in all patients except one, a patient with total anomalous systemic venous connection whose diagnosis was made on the basis of cardiac catheterization. During the study period, several neonates presented to our center but did not undergo surgical intervention because of logistic constraints, financial constraints, or both or because of family preferences; these patients were not included in the analysis.

\section{Outcome Variables, Definitions, and Determinants}

The main outcome variables analyzed included mortality, hospital stay, and postoperative bloodstream infection. The causes of mortality were determined from hospital records. Patients were divided into 2 eras, before and after January 1, 2002, for the purpose of analysis in accordance with the evolution of the neonatal cardiac surgery program at our center. Mortality was defined as in-hospital death or death within 30 days of cardiac surgery. Prolonged hospital stay was defined as a hospital stay of longer than 10 days after cardiac surgery. A positive blood culture was required to establish bloodstream infection (preoperative or postoperative). Wound infection was defined as superficial if the sternum was stable and deep if there was bony involvement and sternal instability. ${ }^{21}$ Transport to the hospital was classified as monitored if the infant was transported in an ambulance accompanied by a doctor/ paramedic staff and unmonitored if the family brought the patient without monitoring or professional assistance. Table 1 summarizes all the variables analyzed as possible determinants of outcome after neonatal cardiac surgery.

\section{Protocols for Neonatal Cardiac Care}

Prostaglandin $\mathrm{E}_{1}$ infusion was started in patients with duct-dependent pulmonary lesions if the systemic arterial oxygen saturation was less than $70 \%$ or if a metabolic acidosis existed. We routinely performed a balloon atrial septostomy in newborns with D-transposition of the great arteries and intact ventricular septum if the patent foramen ovale was restrictive in the presence of low systemic arterial oxygen saturation. The decision to start preoperative 


\section{TABLE 1. Variables included in the analyses}

\begin{tabular}{|c|c|}
\hline Preoperative variables & $\begin{array}{l}\text { Age at diagnosis and operation, sex, } \\
\text { cardiac diagnosis, monitored or } \\
\text { unmonitored transport, } \\
\text { preoperative sepsis (total } \\
\text { leukocyte count, platelet count, } \\
\text { blood culture), use of preoperative } \\
\text { antibiotics, oxygen saturation, use } \\
\text { of prostaglandin } \mathrm{E}_{1} \text {, and } \\
\text { preoperative catheter-based } \\
\text { procedures }\end{array}$ \\
\hline Intraoperative variables & $\begin{array}{l}\text { Use of cardiopulmonary bypass and } \\
\text { total circulatory arrest with } \\
\text { respective durations, modified } \\
\text { ultrafiltration, sternum left open/ } \\
\text { closed after surgery }\end{array}$ \\
\hline Postoperative variables & $\begin{array}{l}\text { Mortality, duration of ventilation, } \\
\text { intensive care unit and hospital } \\
\text { stay, re-intubation, chest re- } \\
\text { exploration, days to delayed } \\
\text { sternal closure, presence of } \\
\text { sepsis or wound infection, use of } \\
\text { antibiotics, and exchange } \\
\text { transfusion for sepsis }\end{array}$ \\
\hline
\end{tabular}

antibiotics was made on clinical grounds (presence of fever, increased leukocyte count, and increased C-reactive protein level). The use of modified ultrafiltration ${ }^{22}$ began after 2002, and the decision to perform delayed sternal closure was left to the discretion of the operating surgeon. Sternal closure was performed as soon as hemodyanamic stability and adequate diuresis were achieved. ${ }^{23}$ Ventilatory management and weaning from mechanical ventilation were performed as per standard guidelines. ${ }^{24} \mathrm{Re}-$ intubation was performed when clinically indicated. Doublevolume exchange transfusion was performed for postoperative sepsis that appeared unresponsive to intravenous antibiotics, especially when accompanied by low leukocyte counts (total leukocyte count $<4000 / \mathrm{mm}^{3}$ or neutrophil count $<1500 / \mathrm{mm}^{3}$ ) or platelet counts $\left(<20,000 / \mathrm{mm}^{3}\right){ }^{25}$ Exchange transfusion was performed with fresh whole blood $(<24$ hours old) as per standard recommendations for neonatal exchange transfusions. ${ }^{25}$ Permission to review hospital records was obtained from the medical records division of the institution.

\section{Statistical Analysis}

The main outcome variables (early mortality, prolonged hospital stay, and postoperative bloodstream infection) were stratified into 2 groups for the purpose of analysis. For continuous variables, the independent-samples $t$ test was performed, and discrete variables were analyzed by using the $\chi^{2}$ test. All variables found to be significant on the univariate analysis were then stratified into groups with a reference group for comparison and entered into a logistic regression model for multivariate analysis. The results of the multivariate analysis were expressed as an odds ratio for risk of association with the outcome variables. Comparison of prevalence
TABLE 2. Baseline characteristics

\begin{tabular}{lc}
\hline Age at diagnosis $(\mathrm{d})^{*}$ & $10.5 \pm 8.1$ \\
Age at operation $(\mathrm{d})^{*}$ & $18.4 \pm 8.3$ \\
Weight at operation $(\mathrm{kg})^{*}$ & $2.8 \pm 0.4$ \\
Monitored transport (\%) & 41.8 \\
Preoperative prostaglandin use (\%) & 13.6 \\
Balloon atrial septostomy (\% of D-TGA) & 57.7 \\
Preoperative blood stream infection (\%) & 2.7 \\
Preoperative antibiotic use (\%) & 4.2 \\
Year of cardiac surgery & No. of patients (\%) \\
January 1999-December 2001 & $98(29.7)$ \\
January 2002-April 2006 & $232(70.3)$ \\
\hline
\end{tabular}

$D$-TGA, D-transposition of the great arteries. *Data are presented as means \pm standard deviation.

of mortality, postoperative bloodstream infection, surgical site infection, and proportion of patients having prolonged hospital stay between the 2 eras (ie, before and after 2002) was performed by using $\chi^{2}$ analysis.

\section{Results}

\section{Baseline Characteristics}

A total of 330 neonates underwent corrective or palliative cardiac surgery at our center during the study period from January 1999 through April 2006. The baseline characteristics of all 330 patients are summarized in Table 2. Of the total, 98 had operations before January 1, 2002, and the remaining 232 had operations thereafter. Male subjects comprised $65.2 \%$ of the entire group. A total of $138(41.8 \%)$ patients were transferred to our institution by medical/ trained paramedic personnel. Prostaglandin $\mathrm{E}_{1}$ was administered to $45(13.6 \%)$ infants with duct-dependent circulations before surgical intervention, and 67 (57.7\%) of the neonates with D-transposition of great arteries underwent preoperative balloon atrial septostomy.

Of the total 330 operations, 208 (63\%) were performed with cardiopulmonary bypass (CPB). Details of the cardiac diagnoses and operations are presented in Table 3. There were $248(75.2 \%)$ corrective procedures and $82(24.8 \%)$ palliative procedures (eg, aortopulmonary shunt or pulmonary artery banding). The mean duration of CPB was $236.2 \pm$ 90.3 minutes. Total circulatory arrest was used in 93 $(28.2 \%)$ of the patients. Modified ultrafiltration was performed in 115 (34.8\%) of the patients before completing CPB. The sternum was electively left open after the operation in $143(43.3 \%)$ patients. After the operation, 18 $(4.2 \%)$ patients required chest re-exploration, and 40 $(9.3 \%)$ required reintubation in the ICU. Mean \pm standard deviation duration of mechanical ventilation was $3.6 \pm 3.3$ days, duration of inotropic use was $4.1 \pm 3.3$ days, ICU stay was $7.9 \pm 5$ days, and hospital stay was $15.4 \pm 10.3$ days. 
TABLE 3. Details of cardiac diagnosis and operations performed and mortality

\begin{tabular}{|c|c|c|c|c|}
\hline \multirow[t]{3}{*}{ Cardiac diagnosis and operation } & \multicolumn{4}{|c|}{$\begin{array}{l}\text { Mortality and total no. of } \\
\text { operations }\end{array}$} \\
\hline & \multicolumn{2}{|c|}{ Before 2002} & \multicolumn{2}{|c|}{ After 2002} \\
\hline & Mortality & Total & Mortality & Tota \\
\hline $\begin{array}{l}\text { D-transposition of the great } \\
\text { arteries }\end{array}$ & 7 & 22 & 0 & 94 \\
\hline $\begin{array}{l}\text { Total anomalous pulmonary } \\
\text { venous connection }\end{array}$ & 0 & 6 & 4 & 42 \\
\hline VSD & 2 & 6 & 0 & 11 \\
\hline Patent ductus arteriosus & 1 & 8 & 1 & 11 \\
\hline Coarctation of aorta & 2 & 12 & 0 & 21 \\
\hline VSD closure + coarctation repair & 0 & 2 & 1 & 2 \\
\hline Aortopulmonary window & 0 & 2 & 0 & 7 \\
\hline Modified Blalock-Taussig shunt & 6 & 35 & 4 & 33 \\
\hline $\begin{array}{l}\text { Coarctation repair }+ \text { pulmonary } \\
\text { artery banding }\end{array}$ & 0 & 2 & 0 & 0 \\
\hline Pulmonary artery banding & 0 & 0 & 0 & 5 \\
\hline Interrupted aortic arch repair & 1 & 3 & 0 & 1 \\
\hline $\begin{array}{l}\text { Anomalous left coronary from } \\
\text { pulmonary artery }\end{array}$ & 0 & 0 & 0 & 2 \\
\hline Hemitruncus repair & 0 & 0 & 0 & 1 \\
\hline $\begin{array}{l}\text { Total anomalous systemic venous } \\
\text { connection }\end{array}$ & 0 & 0 & 0 & 1 \\
\hline $\begin{array}{l}\text { Hemianomalous pulmonary } \\
\text { venous connection }\end{array}$ & 0 & 0 & 0 & 1 \\
\hline Total & 19 & 98 & 10 & 232 \\
\hline
\end{tabular}

VSD, Ventricular septal defect.

\section{Outcome Variables}

Overall mortality was $8.8 \%$ (29 patients). The mortality decreased from $21.4 \%$ in the period before January 1, 2002, to $4.3 \%$ in the period after January 1, $2002(P<.0001)$. Although the number of operations per year increased significantly after 2002, the mortality rate decreased (Figure 1).

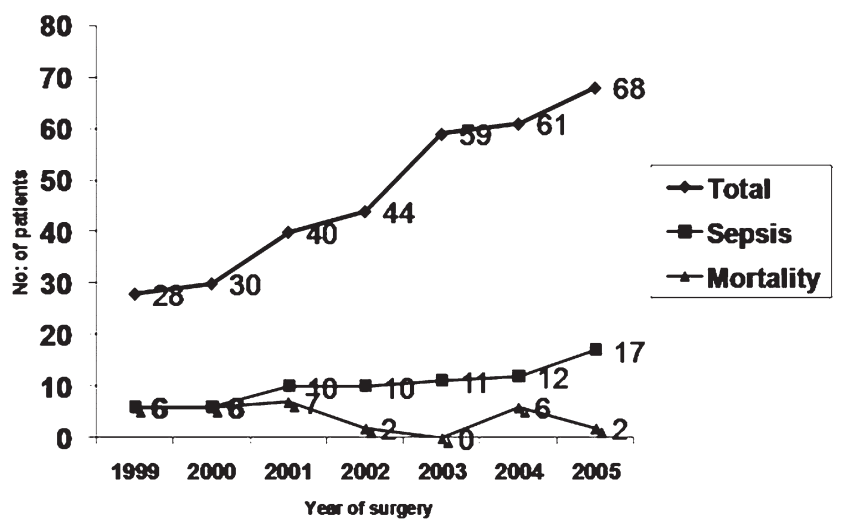

Figure 1. Total number of operations, mortality, and bloodstream infection (sepsis) by year.

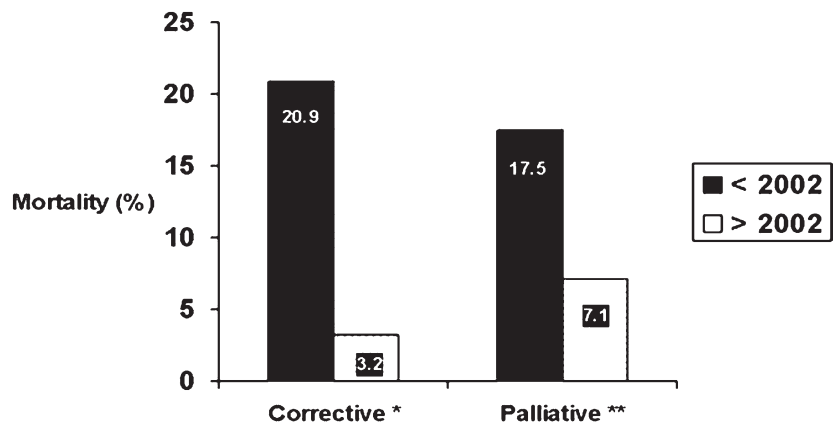

Figure 2. Mortality for corrective and palliative procedures before and after 2002. ${ }^{*} P<.001 ;{ }^{*} P<.05$.

The decrease in mortality after January 1, 2002, was particularly significant for corrective operations (Figure 2). Table 3 shows mortality for individual surgical procedures before and after January 1, 2002. The leading causes of mortality included postoperative bloodstream infection $(\mathrm{n}=$ 9), low cardiac output state $(\mathrm{n}=9)$, pulmonary hypertensive complications $(\mathrm{n}=3)$, and miscellaneous causes $(\mathrm{n}=8)$. The median day of mortality was postoperative day 1 (range, 0-18 days).

Seventy (21.2\%) patients had postoperative bloodstream infection, and 55 (12.7\%) patients had postoperative surgical site infection. The prevalence of postoperative bloodstream infection was not significantly different for the 2 time periods (17.3\% before 2002 vs $22.8 \%$ after $2002, P=$ .33). However, there was a significant increase in the prevalence of surgical site infection in the more recent period ( $9.1 \%$ before 2002 vs $19.8 \%$ after $2002, P=.027$ ). A total of $121(28.2 \%)$ newborns received intravenous antibiotics for bloodstream infection, surgical site infection, or both, and $23(5.4 \%)$ underwent exchange transfusion for severe postoperative sepsis. Prolonged hospital stay ( $>10$ days) was required in $61.2 \%$ of the patients, and the proportion of patients requiring prolonged hospital stay was significantly higher in the more recent period $(38.7 \%$ before 2002 vs $61.6 \%$ after $2002, P \leq .001)$.

There were only 5 premature neonates in the total study population. Two of these 5 died after surgical intervention. Eleven patients had a weight at the time of the operation of less than $2 \mathrm{~kg}$, with the smallest weighing $1.9 \mathrm{~kg}$. Because of the small number of premature/low birth weight patients, we did not attempt to perform a separate analysis for these patients.

Table 4 summarizes the variables significantly associated with mortality, prolonged hospital stay, and postoperative bloodstream infection on multivariate logistic regression analysis. 
TABLE 4. Multivariate logistic regression analysis of predictors of outcome

\begin{tabular}{lccc}
\hline & Odds ratio & 95\% Cl & P value \\
\hline Mortality (predictive power: 92.7\%) & & & \\
Era before January 2002 & 5.5 & $2.2-13.6$ & .0001 \\
Use of exchange transfusion & 14.9 & $4.7-47.6$ & .0001 \\
$\quad$ postoperatively & & & \\
Use of preoperative antibiotics & 5.6 & $1.3-25.1$ & .014 \\
Age group $<7$ d & 3.8 & $1.3-11.3$ & .019 \\
Prolonged hospital stay (predictive power: & $78.3 \%$ ) & \\
Delayed sternal closure & 3.6 & $1.8-7.4$ & .0001 \\
Use of CPB & 2.8 & $1.4-5.8$ & .004 \\
Postoperative use of antibiotics & 4.4 & $2.0-9.3$ & .0001 \\
Reintubation & 12.1 & $2.4-60.6$ & .002 \\
Surgical site infection & 13.8 & $1.7-111.1$ & .015 \\
Postoperative blood stream infection (predictive power: & $92.4 \%)$ \\
Reintubation & 7.7 & $3.6-16.6$ & .0001 \\
Surgical site infection & 4.1 & $2.1-7.9$ & .0001 \\
Use of CPB & 2.0 & $1.1-4.0$ & .04 \\
\hline
\end{tabular}

$\mathrm{Cl}$, Confidence interval; $C P B$, cardiopulmonary bypass.

\section{Discussion}

We report the early outcomes of cardiac surgery in a cohort of 330 consecutive neonates in a tertiary care pediatric cardiac program in southern India. The infrastructure limitations that exist in our program are typical of centers performing pediatric cardiac surgery in the developing world. The overall mortality for all neonatal corrective and palliative cardiac surgical procedures was $8.8 \%$. There was a significant reduction in postoperative mortality from $21.4 \%$ before 2002 to $4.3 \%$ after $2002(P<.0001)$. The reduction in mortality was particularly striking for corrective operations $(20.9 \%$ before 2002 to $3.2 \%$ after $2002, P<$ .0001 , Figure 2), and this compares favorably with outcomes reported from developed countries. $3,5,7,10,12$ The reduction in mortality was paralleled by a significant increase in the annual number of surgical procedures (Figure 1). However, surgical site infections were more common, and the duration of hospital stay was longer after 2002.

On multivariate analysis, the earlier period of surgical intervention (before 2002), age of less than 7 days, need for preoperative antibiotics, and requirement of exchange transfusion for postoperative sepsis emerged as significant predictors of mortality. Improvement in outcomes with growing experience has been reported by other centers and is associated with the initial learning curve of new programs. ${ }^{3,5}$ Our study design does not allow us to determine the specific reasons that contributed to improvements in outcomes. It is possible that a number of factors might have contributed.

The adverse effect of age of less than 7 days at operation on mortality is a significant finding. Although the propor- tion of palliative procedures was greater in younger patients (47.5\% of operations performed in the first week of life were palliative compared with $21.3 \%$ performed after 1 week), the type of operation (palliative vs corrective) did not affect mortality on multivariate analysis. In a previous report on neonatal Blalock-Taussig shunts, we reported an immediate postoperative mortality of $10 \% .^{26}$ It was a routine practice in our center to perform corrective operations, such as the arterial switch operation, if feasible, in the second week of life after initial stabilization with interventions such as a balloon atrial septostomy.

The other predictors of mortality were directly or indirectly related to infectious complications. Preoperative bloodstream infections were present in $2.7 \%$ of patients, and $4.2 \%$ required preoperative antibiotics. These rates remained constant throughout the study period and could have resulted from delays in diagnosis and referral and frequent $(60 \%)$ unmonitored transports. ${ }^{17}$

Postoperative bloodstream infections and surgical wound infections were observed in approximately one quarter of our patients. Double-volume exchange transfusion (performed for neonates with multidrug-resistant gram-negative sepsis with neutropenia and severe thrombocytopenia) was the other predictor for mortality in our study. This could be a reflection of the overall suboptimal physiologic state of the patients caused by severe sepsis because these patients tended to have more hemodyanamic instability and multiorgan dysfunction. We think the underlying indication for exchange transfusion, rather than the procedure itself, produced the increased risk of mortality.

Despite a significant reduction in mortality, the occurrence of bloodstream infections did not decrease during the study period. Furthermore, the frequency of surgical site infections and the proportion of patients requiring a hospital stay of longer than 10 days increased significantly after 2002. Analysis of predictors of prolonged hospital stay revealed use of $\mathrm{CPB}$, delayed sternal closure, postoperative antibiotics, presence of wound infection, and reintubation as significant factors. Delayed sternal closure as a strategy was adopted when the patient showed cardiopulmonary compromise during an attempt at primary sternal closure. ${ }^{23}$ Such patients generally required longer and more intense ventilatory and inotropic support and were more vulnerable to infectious complications caused by an open chest wound and the presence of indwelling catheters for longer periods of time. ${ }^{23}$ Reuse of disposable equipment was not practiced in the operating room (except for aortic and venous cannulae, which were reused after sterilization with ethylene oxide) or in the postoperative unit; therefore it is unlikely that contaminated equipment could have contributed to the high incidence of infectious complications. ${ }^{16}$

The results of this study are of particular relevance to new pediatric cardiac programs that are being established in 
emerging economies. Many countries in the developing world are now experiencing a demographic shift in pediatric diseases as their human development indices improve. As neonatal and infant mortality from readily preventable conditions decrease, CHDs are likely to require increasing attention. Because of resource limitations and several logistic issues, there are many challenges to establishing and maintaining a neonatal cardiac surgery program in these countries. Our results show that with experience, in-hospital mortality, especially for corrective surgical interventions, can be reduced to levels comparable with those of developed countries. Few published reports on the outcomes of neonatal cardiac surgery are available from the developing world. ${ }^{18,19,26}$ These studies relate to specific conditions and not to the full spectrum of CHD in neonates.

Our study indicates that despite a reduction in mortality, morbidity from infectious complications appears to be considerable, and this might be a major stumbling block for further progress. Development and strict enforcement of infection-control policies (eg, hand washing before any direct patient contact, sterile technique for invasive catheter insertion, and early removal of invasive catheters) is therefore highly relevant. ${ }^{27}$ Pediatric cardiac programs in developing countries that attempt neonatal cardiac surgery should include attention to infection-control policies and procedures when establishing their clinical practice. Most infection-control practices can be introduced at relatively low cost and have the potential to improve outcomes significantly.

There are inherent limitations resulting from the retrospective nature of our study. We were not able to analyze and adjust the risk factors with respect to underlying organ function, and objective measures, such as blood lactate level or mixed venous oxygen saturation, were not analyzed as markers of hemodyanamic compromise. Another important limitation is the exclusion of highly complex lesions, such as hypoplastic left heart syndrome and severe Ebstein anomaly; excluding these would be expected to improve the outcomes reported in this study. ${ }^{28,29}$ During the study period, several neonates were admitted to our center who did not undergo operations because of logistic issues or reluctance on the part of their families; this also could have introduced a selection bias.

\section{Conclusions}

With increasing experience, neonatal cardiac surgery can be performed with excellent outcomes in developing countries that have limited infrastructure and resources. Infectious complications account for considerable morbidity and mortality, and improvements in infection-control practices are likely to improve surgical outcomes further in this challenging age group.

\section{References}

1. Wernovsky G, Mayer JE Jr, Jonas RA, Hanley FL, Blackstone EH, Kirklin JW, et al. Factors influencing early and late outcome of the arterial switch operation for transposition of the great arteries. $J$ Thorac Cardiovasc Surg. 1995;109:289-301.

2. Wernovsky G, Wypji D, Jonas RA, Mayer Jr JE, Hanley FL, Hickey $\mathrm{PR}$, et al. Postoperative course and hemodynamic profile after the arterial switch operation in neonates and infants. A comparison of low flow cardiopulmonary bypass and circulatory arrest. Circulation. 1995;92:2226-35.

3. Norwood WI, Dobell AR, Freed MD, Kirklin JW, Blackstone EH Intermediate results of the arterial switch repair. A 20-institution study. J Thorac Cardiovasc Surg. 1988;96:854-63.

4. Culbert EL, Ashburn DA, Cullen-Dean G, Joseph JA, Williams WG, Blackstone EH, et al. for the Congenital Heart Surgeons Society. Quality of life in children after repair of transposition of great arteries. Circulation. 2003;108:857-62.

5. Jacobs JP, Mavroudis C, Jacobs ML, Lacour-Gayet FG, Tchervenkov CI, Gaynor JW, et al. Lessons learned from the data analysis of the second harvest (1998-2001) of the Society of Thoracic Surgeons (STS) Congenital Heart Surgery Database. Eur J Cardiothorac Surg. 2004; 26:18-37.

6. Rossi AF, Seiden H, Sadeghi AM, Nguyen KH, Quintana CS, Gross $\mathrm{RP}$, et al. The outcome of cardiac operations in infants weighing two kilograms or less. J Thorac Cardiovasc Surg. 1998;116:28-32.

7. Reddy VM, McElhinney DB, Sagrado T, Parry AJ, Teitel DF, Hanley FL. Results of 102 cases of complete repair of congenital heart defects in patients weighing 700 to 2500 grams. J Thorac Cardiovasc Surg. 1999;117:324-31.

8. Hennein HA, Mosca RS, Urcelay G, Crowley DC, Bove EL. Intermediate results after complete repair of tetralogy of Fallot in neonates. J Thorac Cardiovasc Surg. 1995;109:332-44.

9. Conte S, Layour-Gayet F, Serraf A, Uva MS, Bruniaux J, Touchot A, et al. Surgical management of neonatal coarctation. J Thorac Cardiovasc Surg. 1995;109:663-75.

10. Bove T, Francois K, De Groote K, Suys B, De Wolf D, Verhaaren H, et al. Outcome analysis of major cardiac operations in low weight neonates. Ann Thorac Surg. 2004;78:181-7.

11. Rodefeld MD, Hanley FL. Neonatal truncus arteriosus repair: surgical techniques and clinical management. Semin Thorac Cardiovasc Surg Pediatr Card Surg Ann. 2002;5:212-7.

12. Shen I, Giacomuzzi C, Ungerleider RM. Current strategies for optimizing the use of cardiopulmonary bypass in neonates and infants. Ann Thorac Surg. 2003;75(suppl):S729-34.

13. Mahle WT, Forbess JM, Kirshborn PM, Cuadrado AR, Simsic JM, Kanter KR. Cost-utility analysis of salvage cardiac extracorporeal membrane oxygenation in children. $J$ Thorac Cardiovasc Surg. 2005; 129:1084-90.

14. Twedell JS, Spray TL. Newborn heart surgery: reasonable expectations and outcomes. Pediatr Clin North Am. 2004;51:1611-23.

15. Culbert EL, Ashburn DA, Cullen-Dean G, Joseph JA, Williams WG, Blackstone EH, et al. for the Congenital Heart Surgeons Society. Quality of life children after repair of transposition of great arteries. Circulation. 2003;108:857-62.

16. Kumar RK, Tynan MJ. Catheter interventions for congenital heart diseases in third world countries. Pediatr Cardiol. 2005;26:241-9.

17. Brown KL, Ridout DA, Hoskote A, Verhulst L, Ricci M, Bull C. Delayed diagnosis of congenital heart disease worsens preoperative condition and outcome of surgery in neonates. Heart. 2003;89:436-41.

18. Choudhary SK, Bhan A, Sharma R, Mathur A, Airan B, Saxena A, et al. Repair of total anomalous pulmonary venous connection in infancy: experience from a developing country. Ann Thorac Surg. 1999;68:155-9.

19. Saxena A, Sharma M, Kothari SS, Juneja R, Reddy SC, Sharma R, et al. Prostaglandin E1 in infants with congenital heart disease: Indian experience. Indian Pediatr. 1998;35:1063-9.

20. O'Grady NP, Alexander M, Dellinger EP, Gerberding JL, Heard SO, Maki DG, et al. Guidelines for the prevention of intravascular catheterrelated infections. Centers for Disease Control and Prevention. MMWR Recomm Rep. 2002;51:1-29. 
21. Garner J, Jarvis W, Emori T, Horan T, Huges J. CDC definitions for nosocomial infections 1988. Am J Infect Control. 1988;16:128-40.

22. Naik SK, Knight A, Elliot MJ. A prospective, randomized study of a modified technique of ultra filtration during pediatric open-heart surgery. Circulation. 1991;84(suppl III):422-31.

23. Hakimi M, Walters HL, Pinsky WW, Gallagher MJ, BS, Lyons JM. Delayed sternal closure after neonatal cardiac operations. $J$ Thorac Cardiovasc Surg. 1994;107:925-33.

24. Zucker HA. The airway and mechanical ventilation. In: Chang AC, Hanley FL, Wernovsky G, editors. Pediatric cardiac intensive care. Baltimore: Williams and Wilkins; 1998. p. 95-106.

25. Vain NE, Mazlumian JR, Swarner OW, Cha CC. Role of exchange transfusion in the treatment of severe septicemia. Pediatrics. 1980;66:693-7.
26. Sivakumar K, Shivaprakasha K, Rao SG, Kumar RK. Operative outcome and intermediate follow-up of neonatal Blalock-Taussig shunts. Indian Heart J. 2001;53:66-70.

27. McGee DC, Gould MK. Preventing complications of central venous catheterization. $N$ Engl J Med. 2003;348:1123-33.

28. Tweddell JS, Hoffman GM, Mussatto KA, Fedderly RT, Berger S, Jaquiss RD, et al. Improved survival of patients undergoing palliation of hypoplastic left heart syndrome: lessons learned from 115 consecutive patients. Circulation. 2002;106(suppl 1):182-9.

29. Knott-Craig CJ, Overholt ED, Ward KE, Razook JD. Neonatal repair of Ebstein's anomaly: indications, surgical technique, and medium term follow up. Ann Thorac Surg. 1999;67: $1137-41$

\section{Notice of Retraction}

The Editor of the Journal regrets to inform our readers that the article Malagon I, Onkenhout W, Klok M, Linthorst L, van der Poel PFH, Bovill JG, and Hazekamp MG. Dexamethasone reduces gut permeability in pediatric cardiac surgery. J Thorac Cardiovasc Surg 2005;130:265-271 has been retracted.

Subsequent to its publication, the authors indicated that the study was not prospectively randomized as stated in the Methods section. Upon independent review as an observational study, it was concluded that the findings were underpowered to allow the conclusions drawn with adequate certainty. 